\title{
One dose of SARS-CoV-2 vaccine exponentially increases antibodies in individuals who have recovered from symptomatic COVID-19
}

\author{
Riccardo Levi, ${ }^{1}$ Elena Azzolini, ${ }^{1,2}$ Chiara Pozzi, ${ }^{2}$ Leonardo Ubaldi, ${ }^{1}$ Michele Lagioia, ${ }^{2}$ Alberto Mantovani, ${ }^{1,2,3}$ and Maria Rescigno ${ }^{1,2}$ \\ 'Department of Biomedical Sciences, Humanitas University, Milan, Italy. ${ }^{2} I R C S S$ Humanitas Research Hospital, Milan, Italy. ${ }^{3}$ The William Harvey Research Institute, Queen Mary University of London, \\ London, United Kingdom.
}

\begin{abstract}
BACKGROUND. The COVID-19 vaccines currently in use require 2 doses to achieve optimal protection. Currently, there is no indication as to whether individuals who have been exposed to SARS-CoV-2 should be vaccinated, or whether they should receive 1 or 2 vaccine doses.
\end{abstract}

\begin{abstract}
METHODS. We tested the antibody response developed after administration of the Pfizer/BioNTech vaccine in 124 health care professionals, of whom $\mathbf{5 7}$ had a previous history of SARS-CoV-2 exposure with or without symptoms.

RESULTS. Postvaccine antibodies in SARS-CoV-2-exposed individuals increased exponentially within $\mathbf{5}$ to 18 days after the first dose compared to naive subjects $(P<0.0001)$. In a multivariate linear regression $(L R)$ model we showed that the antibody response depended on the IgC prevaccine titer and on the exposure to SARS-CoV-2. In symptomatic SARS-CoV-2-exposed individuals, IgG reached a plateau after the second dose, and those who voluntarily refrained from receiving the second dose $(n=7)$ retained their antibody response. Gastrointestinal symptoms, muscle pain, and fever markedly positively correlated with increased IgG responses. By contrast, all asymptomatic/paucisymptomatic and unexposed individuals showed an important increase after the second dose.
\end{abstract}

CONCLUSION. One vaccine dose is sufficient in symptomatic SARS-CoV-2-exposed subjects to reach a high titer of antibodies, suggesting no need for a second dose, particularly in light of current vaccine shortage.

TRIAL REGISTRATION. ClinicalTrials.gov NCT04387929.

FUNDING. Dolce \& Gabbana and the Italian Ministry of Health (Ricerca corrente).

\section{Introduction}

An unprecedented effort in vaccine development has led to the emergency approval of the first SARS-CoV-2 vaccine by the US Food and Drug Administration around 10 months from the initial reported COVID-19 cases. It is the Pfizer/BioNTech vaccine, an mRNA-based vaccine encoding the SARS-CoV-2 full-length spike protein (BNT162b2) (1). The phase 3 clinical trial showed this vaccine to have a very safe profile, an efficacy of $95 \%$ seven days after the second dose of vaccine, and an efficacy of nearly $50 \%$ around 12 days from the first dose (2). The vaccine induces a strong antibody response (also neutralizing) after the first dose in individuals younger than 65 years of age (1). Patients older than 65 years require the 2 doses to generate a good anti-

\section{Related Commentary: https://doi.org/10.1172/JCl150135}

Authorship note: RL, EA, CP, and LU contributed equally to this work

Conflict of interest: The authors have declared that no conflict of interest exists. Copyright: ( 2021, American Society for Clinical Investigation.

Submitted: March 1, 2021; Accepted: April 26, 2021; Published: June 15, 2021.

Reference information: J Clin Invest. 2021;131(12):e149154.

https://doi.org/10.1172/JCl149154. body response (1). Except for the Johnson \& Johnson adenoviralvectored SARS-CoV-2 vaccine that is administered as a single dose (3), the other emergency-approved COVID-19 vaccines based on mRNA $(1,2,4)$ or adenovirus (5) require a first dose followed by recall immunization. However, there is no indication as to whether individuals previously exposed to SARS-CoV-2 should be vaccinated or how many times they should receive a vaccine. We and others have shown that antibody response increases robustly after the first dose in SARS-CoV-2-exposed individuals (6-9). By contrast, a second dose of vaccine does not seem to further increase the antibody response and may even have a detrimental effect on antigen-specific immune responses (10). Here we analyzed the antibody response in 124 health care professionals (67 naive and 57 SARS-CoV-2-exposed), of whom 117 received 2 doses of vaccine and 7 SARS-CoV-2-exposed subjects received only 1 dose, and correlated this response with their SARS-CoV-2 infection symptoms.

\section{Results}

Antibody response grows exponentially after 1 dose of vaccine in SARSCoV-2-exposed individuals. We tested the antibody response to the S1/S2 antigen in 124 health care professionals after Pfizer/BioNTech vaccination at around 17 days from the first vaccine dose and 
Observational study

(ClinicalTrials.gov NCT04387929)

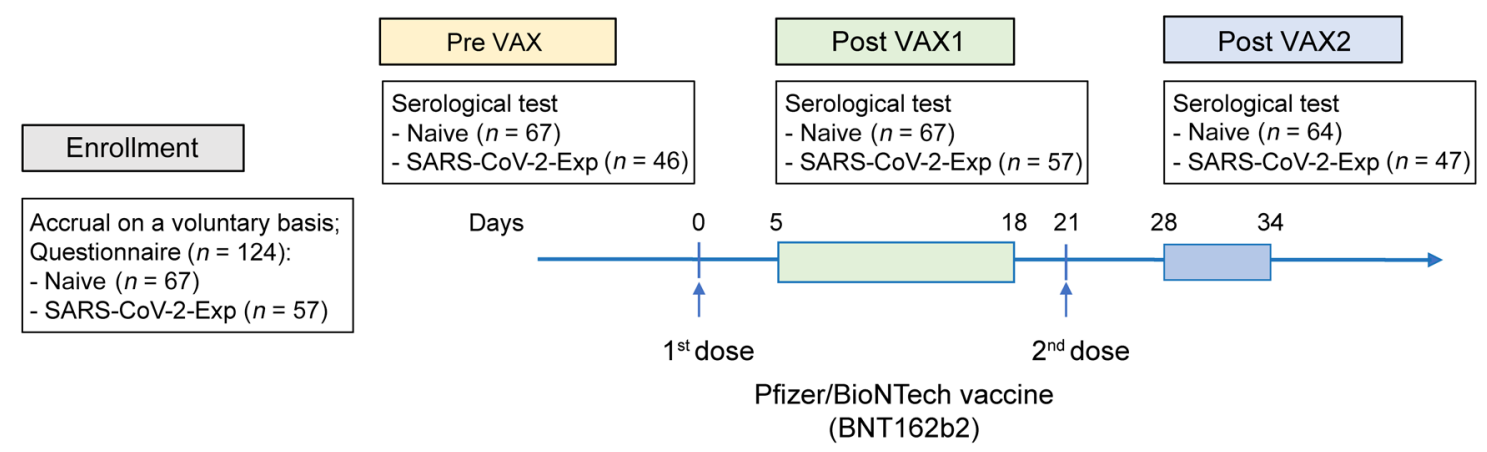

Figure 1. Experimental design. Observational study approved by the international review board of Istituto Clinico Humanitas (ClinicalTrials.gov NCT04387929). Accrual was on a voluntary basis. All participants signed an informed consent and completed a questionnaire indicating a previous history of SARS-CoV-2 exposure and COVID-19-associated symptoms/clinical manifestations (SARS-CoV-2-Exp). This study was conducted at Istituto Clinico Humanitas in which health care and administrative staff were followed for serology before (Pre VAX), after the first dose (Post VAX1), and after the second dose (Post VAX2) of the Pfizer/BioNTech vaccine.

11 days from the second dose (Figure 1 and Supplemental Tables 1 and 2; supplemental material available online with this article; https://doi.org/10.1172/JCI149154DS1). As shown in Supplemental Figure 1, SARS-CoV-2-exposed individuals had significantly higher levels of prevaccine antibodies than nonexposed naive individuals. The prevaccine antibody amount of the SARS-CoV-2exposed population was on average $44.6 \pm 37.7$ while that of the naive population was $3.4 \pm 1(P<0.0001)$ (Supplemental Table 1). At the first time point analyzed after the first dose, the antibody response of SARS-CoV-2-exposed individuals was much higher than that of naive subjects $(P<0.0001)$. They displayed an exponential increase of anti-Spike $1 / 2$ antibody response $(>20$-fold higher than naive individuals, Supplemental Figure 1 and Supplemental Table 1). Interestingly, at around 11 days after the second dose of vaccine the antibody response steadily increased in naive individuals while it reached a plateau in some but not all SARSCoV-2-exposed subjects (Figure 2A and Supplemental Figure 1). The plateau is likely due to a technical limitation as the upper limit of detection of the test $(4000 \mathrm{AU} / \mathrm{mL})$ was reached after a 1:20 dilution of the sample, but further dilutions were not possible as they would lead to unreliable results (Figure 2B).

We investigated the relationship between the amount of $\operatorname{IgG}$ after vaccination with the exposure to SARS-CoV-2, sex, IgG levels prior to vaccination, days from serology, and age in a linear regression analysis. Figure $2 \mathrm{C}$ shows a multivariate linear regression (LR) analysis after the first vaccine dose with a good prediction of the log-transformed IgG amount after the first dose $\left(R^{2}=0.79, F\right.$-statistic $=80.95, P<0.001)$; the most significant positively correlated features were exposure to SARS-CoV-2 (2.12, 95\% CI 1.70-2.55), the value of IgG before vaccination $(0.48,95 \% \mathrm{CI} 0.26-0.69)$, the difference between the date of vaccination and the date of serology after vaccination $(0.54,95 \%$ CI $0.37-0.71)$. The only feature that negatively correlated with IgG titers was age $(-0.15,95 \%$ CI $-0.32-0.01)$, and there was no correlation with sex.

The second multivariate LR analysis after the second vaccine dose (which was set at 21 days) also showed a good prediction of the actual IgG amount after the second dose $\left(R^{2}=0.67, F\right.$-statis- tic $=26.62, P<0.001$, Figure 2D). The most significant positively correlated features were again exposure to SARS-CoV-2 (979.4, 95\% CI 548.8-1409.9), the value of IgG after the first vaccine dose (442.0, 95\% CI 222.5-661.6), the difference between the date of vaccination and the date of serology after vaccine dose 2 (196.9, 95\% CI 40.6-353.2), while there was a negative correlation with the date of vaccine dose 1 (-292.1, 95\% CI -444.9 to -139.3$)$, and there was no significant correlation with age, sex, and the amount of IgG before the first dose of vaccine.

Together these results indicate that SARS-CoV-2-exposed individuals display an exponential increase in antibody response after the first vaccine dose and this correlates with the IgG titers before vaccination, age, and exposure to SARS-CoV-2.

Postvaccine antibody response associates with symptoms. We observed an increase of antibody response in SARS-CoV-2exposed individuals, particularly after the first dose of vaccine. We evaluated whether this response equally increased in symptomatic (with a history of COVID-19) and asymptomatic/paucisymptomatic SARS-CoV-2-exposed individuals. As shown in Figure 3, A and B, the antibody response was higher in symptomatic individuals both after the first dose $(P=0.049)$ and second vaccine dose $(P=0.043)$. However, in 4 of 8 (50\%) symptomatic individuals having IgG titer higher than $1600 \mathrm{AU} / \mathrm{mL}$ after the first vaccine dose, a reduction of antibody production was observed (Figure 3C, red dots and lines). By contrast, none of the symptomatic individuals with IgG levels higher than $1000 \mathrm{AU} / \mathrm{mL}$ who voluntarily decided not to receive the second dose $(n=7)$ displayed any drop in antibody levels at the same time interval of those tested after the second dose $(34.3 \pm 5.5$ days from the first dose), suggesting that the first vaccine dose is sufficient to induce a sustained antibody response (Figure 3, D and E, and Supplemental Tables 3 and 4).

Gastrointestinal symptoms, fever and muscle pain correlate with increased IgG. Having observed that symptomatic SARSCoV-2-exposed individuals displayed higher levels of IgG titers after vaccination, we evaluated which symptoms correlated best with a higher IgG response. We found that gastrointestinal symptoms, fever, and muscle pain all positively correlated with higher 
A
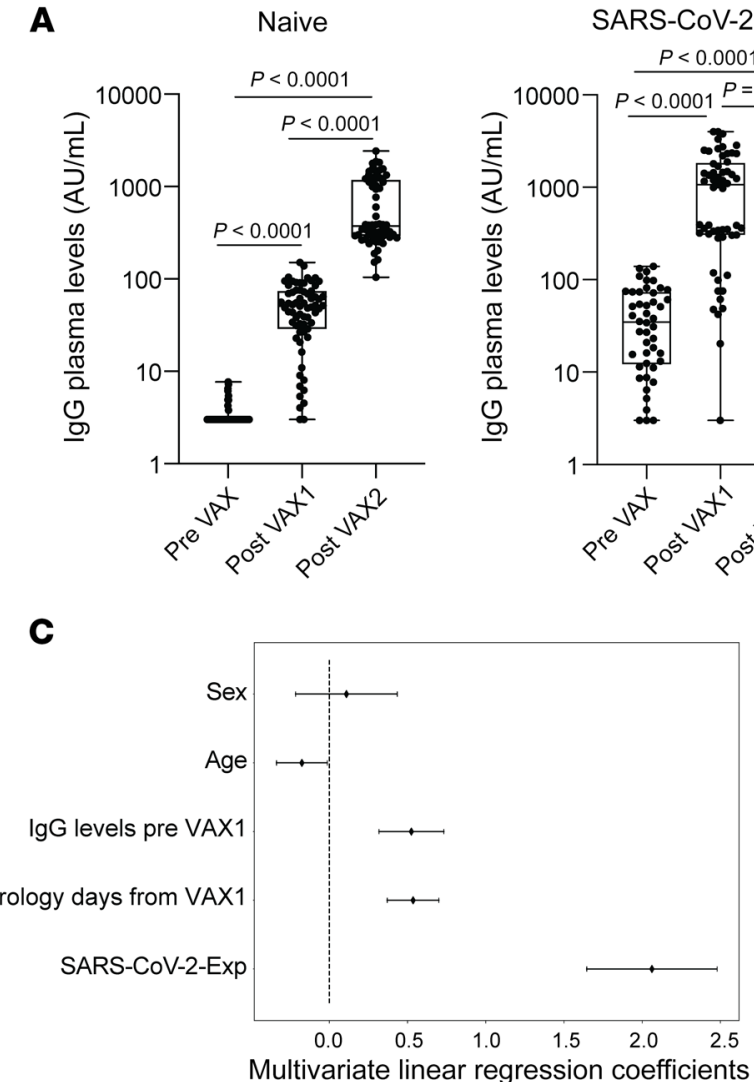

SARS-CoV-2-Exp

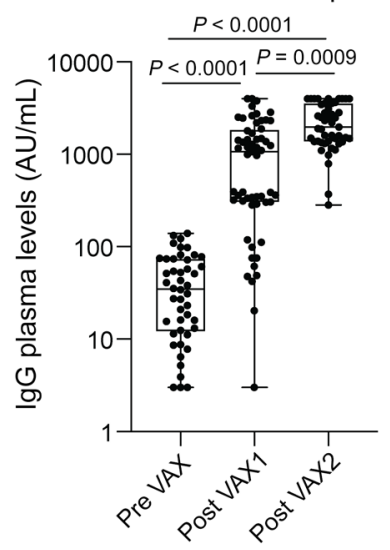

B

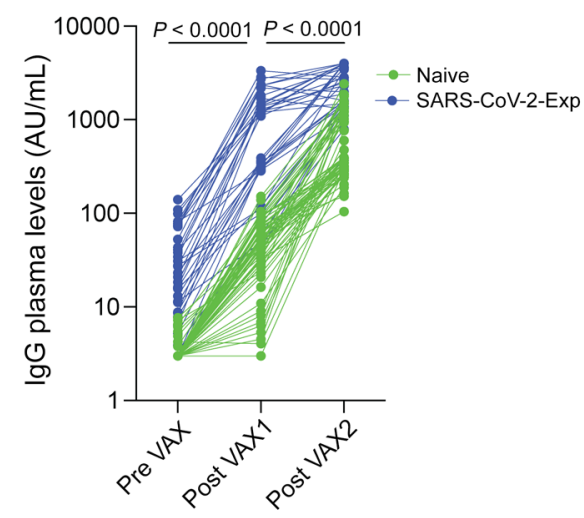

D

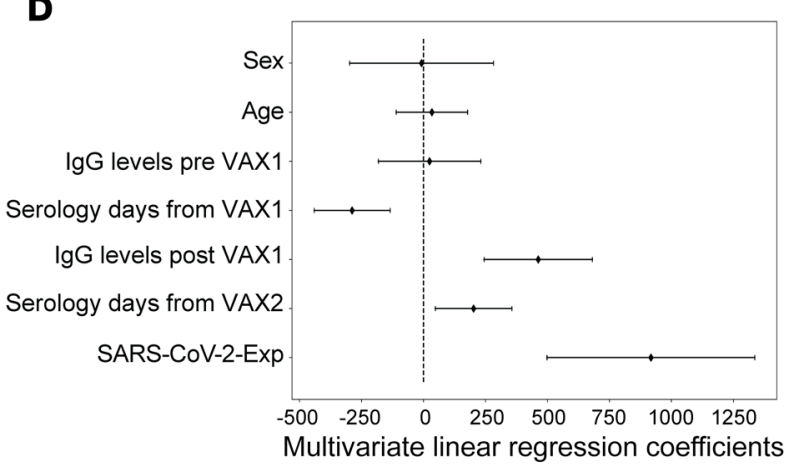

Figure 2. Exponential increase of anti-Spike $1 / 2$ IgG levels after the first dose of vaccine in SARS-CoV-2-exposed subjects. (A) Anti-Spike $1 / 2$ IgG plasma levels before (Pre VAX), after the first dose (Post VAX1) or after the second dose (Post VAX2) of vaccine in naive (Pre VAX, Post VAX1 [ $n=67]$, Post VAX2 $[n=64]$ ) and SARS-CoV-2-exposed individuals (SARS-CoV-2-Exp; Pre VAX [ $n=46]$, Post VAX1 [ $n=57]$, Post VAX2 [ $n=47]$ ). Each dot corresponds to an individual subject. Log scale on y axis. (B) Anti-Spike $1 / 2$ lgG plasma levels in naive $(n=64)$ and SARS-CoV-2-exposed individuals $(n=36)$. Spaghetti plot showing the trends for each individual subject by linked dots. Log scale on y axis. (C) Association between the log-transformed amount of IgC following the first vaccine dose and the clinical variable through multivariate LR (naive $n=67$, SARS-CoV-2-exposed individuals $n=46$ ). The coefficients for the most significant variables $(P<0.05)$ are reported. Dot points represent the mean values of regression coefficients and lines the $95 \%$ Cl. (D) Association between the amount of IgG following the second vaccine dose and the clinical variable through multivariate LR (naive $n=64$, SARS-CoV-2-exposed individuals $n=$ 36). The coefficients for the most significant variables $(P<0.05)$ are reported. Dot points represent the mean values of regression coefficients and lines the $95 \% \mathrm{Cl}$. The box plots (A) show the interquartile range, the horizontal lines show the median values, and the whiskers indicate the minimum-to- maximum range. $P$ values were determined using 2-tailed Kruskal-Wallis test with Dunn's post test (A) or 2-tailed Wilcoxon matched-pairs signed rank test (B).

IgG responses (Supplemental Figure 2 and Supplemental Table 5), even though, as shown earlier, the second vaccine dose did not induce as strong an increase of antibodies as that observed after the first dose. Interestingly, of the 4 subjects who displayed reduction of antibody response, all had more than 5 symptoms; 2 presented with anosmia/dysgeusia, 1 with gastrointestinal symptoms, and 1 with severe pneumonia. Individuals with similar symptoms/clinical manifestations did not show this reduction (at least at this time point).

\section{Discussion}

We have shown that the antibody response of SARS-CoV-2exposed individuals depends on the $\operatorname{Ig} G$ prevaccine titer and positively correlates with the symptoms that the individuals developed during COVID-19. Gastrointestinal symptoms, muscle pain, and fever significantly $(P=0.002, P=0.0012, P=0.0339$, respectively) positively correlated with increased IgG. Of note, we previously observed that anosmia/dysgeusia was associated with an increase of antibodies in the natural infection (11). Here, we found that anosmia/dysgeusia correlated with increased IgG after the second dose of vaccine $(P=0.01)$. While asymptomatic/paucisymptomatic or naive subjects showed a steady increase of antibody response, symptomatic SARS-CoV-2-exposed individuals reached a plateau after the second dose. This was partly due to the upper limit of detection of the test after autodilution (4000 AU/ $\mathrm{mL}$ ). Thus, 1 vaccine dose is sufficient to induce a robust anti-S1/2 antibody response in SARS-CoV-2-exposed subjects. It cannot be excluded that a second vaccine dose may even be detrimental due to hyper-immuneactivation, which could switch-off the immune response due to antigen exhaustion, as it occurs after several viral infections (reviewed in ref. 12). Indeed, we observed that in 4 of the COVID-19 symptomatic cases, the antibody response was even reduced after the second dose, while this was not observed in symptomatic subjects who voluntarily decided not to receive the second dose. These results are in line with a companion report by Francesco Annunziato and colleagues (13), which showed a 
A

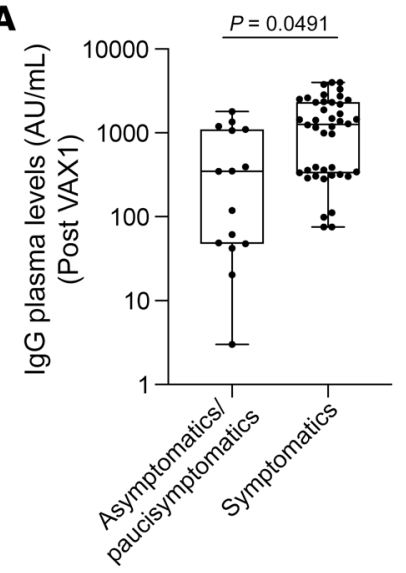

D

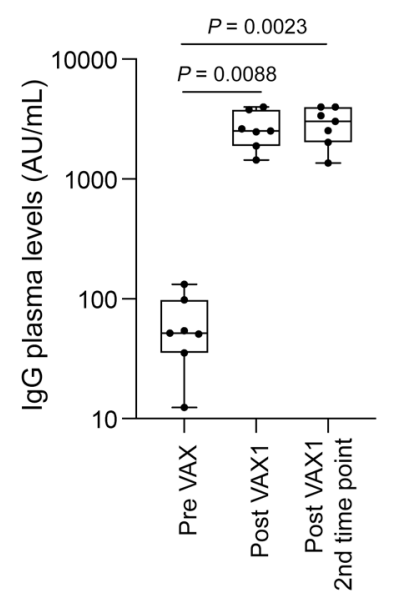

B

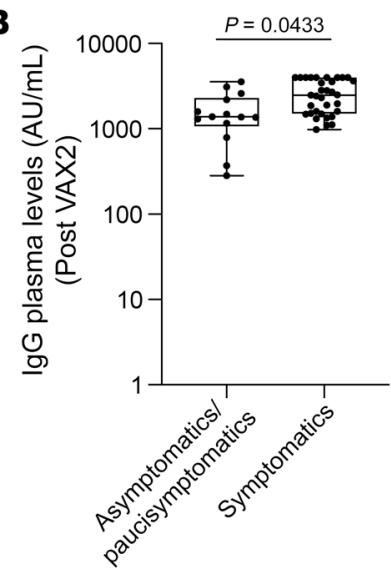

E

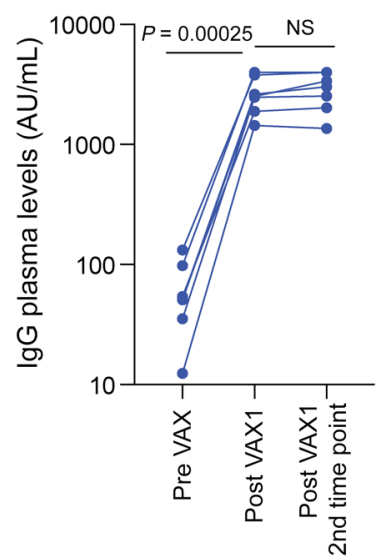

C

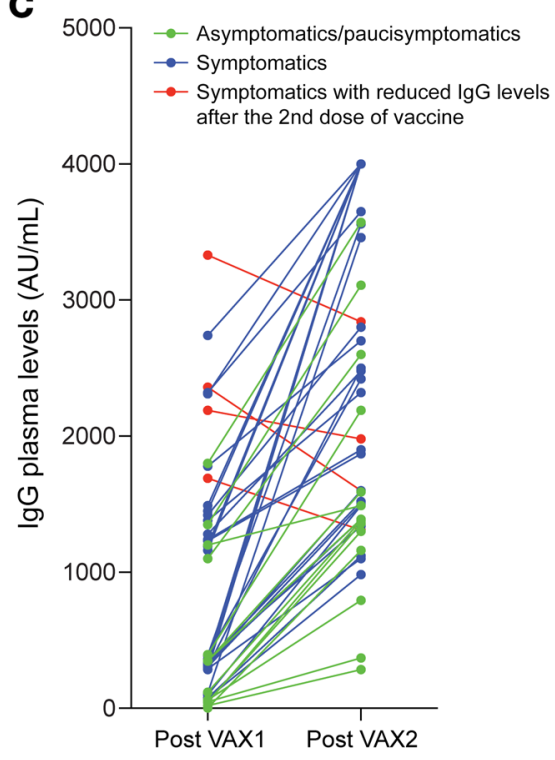

Figure 3. Symptomatic SARS-CoV-2-exposed subjects show higher increase of anti-Spike 1/2 lgG levels after the first dose of vaccine. (A and B) AntiSpike 1/2 IgC plasma levels in asymptomatics/paucisymptomatics $(n=15)$ or symptomatics $(n=42)$ after the first $(\mathbf{A})$ or after the second dose of vaccination (asymptomatics/paucisymptomatics $n=14$, symptomatics $n=33$ ) (B). Each dot corresponds to an individual subject. Log scale on $Y$ axis. (C) Before and after plot (asymptomatics/paucisymptomatics $n=14$, symptomatics $n=33$ ), showing trends for each individual subject by linked dots. (D and $\mathbf{E}$ ) AntiSpike 1/2 IgC plasma levels in symptomatic SARS-CoV-2-exposed individuals $(n=7)$ at around 12 days (Post Vax 1 ) or around 34 days (Post Vax 1 second time point) after the first dose of vaccine (D). Spaghetti plot showing the trends for each individual subject by linked dots (E). Log scale on $y$ axis. The box plots (A, B, and $\mathbf{D}$ ) show the interquartile range, the horizontal lines show the median values, and the whiskers indicate the minimum-to-maximum range. $P$ values were determined using 2-tailed unpaired Kolmogorov-Smirnov test (A and $\mathbf{B}$ ) or 2-tailed Wilcoxon matched-pairs signed rank test (symptomatics post VAX1 vs post VAX 2, $P<0.0001$; asymptomatics/paucisymptomatics post VAX1 vs post VAX2, $P=0.0001)$ (C); 1 -way ANOVA with Tukey's post test (D) or 2-tailed paired Student's $t$ test (E).

reduced number of antigen-specific B cells and neutralizing antibodies after the second vaccine dose, and a recently reported preprint (10). Although the instrument autodiluted the plasma sample when the upper limit of detection was reached, we cannot exclude a prozone effect if the level of IgG after the second vaccine dose was so high to interfere with the assay, resulting in a hook effect and impairment of antibody measure. These results question whether a second shot in symptomatic SARS-CoV-2-exposed subjects is indeed required and suggest its postponement while monitoring antibody response longevity, particularly if antibody titers are higher than $1500 \mathrm{AU} / \mathrm{mL}$ after the first vaccine dose.

At a time of vaccine scarcity, these findings have strong public health implications.

\section{Methods}

IgG determination. For the determination of IgG anti-SARS-CoV-2 the Liaison SARS-CoV-2 S1/S2 IgG assay (DiaSorin) was used (14). The method is an indirect chemiluminescence immunoassay for the determination of anti-S1- and anti-S2-specific antibodies, which allowed us to evaluate even large amounts of plasma IgG after autodilution. Intra- and interassay coefficient of variation are less than $1.9 \%$ and less than $3.7 \%$, respectively. In the data sheet of the test, in a cohort of 304 patients with COVID-19, 97.5\% of the samples above $15 \mathrm{AU} / \mathrm{mL}$ and $86 \%$ of those with IgG greater than $12 \mathrm{AU} / \mathrm{mL}$ resulted positive for neutralizing antibodies with a titer of more than 1:40 in the plaque reduction neutralization test (PRNT). The Liaison assay's performance in comparison to a microneutralization assay is shown in ref. 14. The Liaison serological S1/S2 assay can distinguish between neutralization-positive and -negative samples at cut-offs near $15 \mathrm{AU} / \mathrm{mL}$. Additionally, the data indicate that $92 \%$ of the samples with more than $80 \mathrm{AU} / \mathrm{mL}$ had neutralization titers greater than or equal to 1:80, whereas $87 \%$ of samples with more than $80 \mathrm{AU} / \mathrm{mL}$ had neutralization titers greater than of equal to 1:160. The sensitivity of the test as reported by the manufacturer is $90.4 \%(79.4 \%-95.8 \%)$ and its specificity is $98.5 \%$ (97.5\%-99.2\%). 
Linear regression. To predict the IgG response after 2 doses of SARS-COV-2 vaccine, 2 separate multivariate LR models were developed. Numerical independent variables were standardized ( $z$ score algorithm) in both models, and the IgG amount after the first dose of vaccine, when analyzed as a dependent variable, was log-transformed due to right asymmetry of the distribution. Only for this regression analysis, the subjects without the serological value before vaccination were excluded $(n=11)$. The final number of subjects analyzed in the first dose LR was 113, whereas in the second dose LR was 100.

Statistics. Data were analyzed for normal distribution (Shapiro-Wilk test) before any statistical analyses. Individual values are presented as before-after or spaghetti plots or as box plots showing the interquartile range, median, and minimum-to-maximum whiskers. Statistical significance between 2 groups was determined using 2-tailed paired Student's $t$ test, 2-tailed unpaired Mann-Whitney $U$ test, or 2-tailed Wilcoxon matched-pairs signed rank test, whereas the comparison of multiple groups was carried out using 1-way ANOVA with Tukey's post test or Kruskal-Wallis test followed by Dunn's test. A probability value of $P$ less than 0.05 was considered significant. All statistics and reproducibility information are reported in the figure legends. Data analyses were carried out using GraphPad Prism version 8 and Python version 3.8 with the following libraries: Pandas (version 1.1.4, data wrangling), Scipy (version 1.3.2, statistical analysis), and Statsmodel (version 0.12.1, LR statistical model).

Study approval. We tested the antibody response developed after the Pfizer/BioNTech vaccine (1) in 124 health care professionals, of whom 57 had a previous history of SARS-CoV-2 exposure, as part of an observational study approved by the international review board of Istituto Clinico Humanitas (ClinicalTrials.gov NCT04387929). Accrual was on a voluntary basis. The study was conducted at Istituto Clinico Humanitas, where health care and administrative staff were followed for serology and for any occurring COVID-19-associated symptoms or clinical manifestations every 3 months (15) or after Pfizer/BioNTech vaccination at around 17 days from the first vaccine dose and 11 days from the second dose (Figure 1). All participants signed an informed consent and completed a questionnaire before blood collection. In this study, we analyzed 15 features including, age, sex, IgG levels (before vaccination, after first dose, after second dose), days between serological test and vaccine dose, a previous history of SARS-CoV-2 exposure, COVID-19-associated symptoms (including fever, low grade fever, cough, sore throat and/or runny nose, headache, asthenia, soreness and muscle pain, anosmia/dysgeusia, gastrointestinal symptoms, conjunctivitis, dyspnea, chest pain), and clinical manifestations such as tachycardia and/or pneumonia. We considered asymptomatics subjects without any symptoms; paucisymptomatics individuals who developed 1 or 2 symptoms; and symptomatics individuals with more than 3 symptoms. Clinical manifestations (tachycardia and pneumonia) were always associated with at least other 2 symptoms. For demographics characteristics please refer to Supplemental Table 1.

\section{Author contributions}

RL performed LR analysis. CP performed data analysis and contributed to writing the manuscript. LU contributed to data analysis. EA and ML coordinated the recruitment and sampling of subjects (project administration) and participated in clinical study design. AM conceptualized the study and acquired funding. MR conceived the study, analyzed the data, and wrote the manuscript.

\section{Acknowledgments}

This work was partially supported by a philanthropic donation by Dolce \& Gabbana and by the Italian Ministry of Health (Ricerca corrente). We would like to thank the employees who volunteered to participate to the study, the nurses and personnel who collected the samples, and the laboratory technicians who ran the serological tests. We would also like to thank the Humanitas management and staff, and Patrizia Meroni, who warmly supported this study for the safety of the employees.

Address correspondence to: Maria Rescigno or Alberto Mantovani, Humanitas University, Via Rita Levi Montalcini 4, 20090 Pieve Emanuele, Milan, Italy. Email: maria.rescigno@hunimed. eu (MR); alberto.mantovani@hunimed.eu (AM).
1. Walsh EE, et al. Safety and immunogenicity of 2 RNA-based Covid-19 vaccine candidates. $N$ Engl JMed. 2020;383(25):2439-2450.

2. Polack FP, et al. Safety and efficacy of the BNT162b2 mRNA Covid-19 vaccine. $N$ Engl J Med. 2020;383(27):2603-2615.

3. Sadoff J, et al. Interim results of a phase 1-2a trial of Ad26.COV2.S Covid-19 vaccine [published online January 13, 2021]. N Engl JMed. https:// doi.org/10.1056/NEJMoa2034201.

4. Baden LR, et al. Efficacy and safety of the mRNA-1273 SARS-CoV-2 vaccine. $N$ Engl J Med. 2021;384(5):403-416.

5. Voysey M, et al. Safety and efficacy of the ChAdOx1 nCoV-19 vaccine (AZD1222) against SARSCoV-2: an interim analysis of four randomised controlled trials in Brazil, South Africa, and the UK. Lancet. 2021;397(10269):99-111.

6. Krammer F, et al. Robust spike antibody responses and increased reactogenicity in seropositive individuals after a single dose of SARS-CoV-2 mRNA vaccine [preprint]. https://doi.org/10.1
101/2021.01.29.21250653. Posted on medRxiv February 1, 2021.

7. Saadat S, et al. Single dose vaccination in healthcare workers previously infected with SARSCoV-2. JAMA. 2021;325(14):1467-1469.

8. Abu Jabal K, et al. Impact of age, ethnicity, sex and prior infection status on immunogenicity following a single dose of the BNT162b2 mRNA COVID-19 vaccine: real-world evidence from healthcare workers, Israel, December 2020 to January 2021. Euro Surveill. 2021;26(6):2100096.

9. Levi $\mathrm{R}$, et al. A cautionary note on recall vaccination in ex-COVID-19 subjects [preprint]. https:// doi.org/10.1101/2021.02.01.21250923. Posted on medRxiv February 6, 2021.

10. Samanovic MI, et al. Poor antigen-specific responses to the second BNT162b2 mRNA vaccine dose in SARS-CoV-2-experienced individuals [preprint]. https://doi.org/10.1101/2021.02.0 7.21251311. Posted on medRxiv February 9, 2021. 11. Levi $\mathrm{R}$, et al. The antibody response to SARS-
CoV-2 increases over 5 months in patients with anosmia/dysgeusia [preprint]. https://doi.org/10 $.1101 / 2021.02 .05 .21251219$. Posted on medRxiv February 8, 2021.

12. Yi JS, et al. T-cell exhaustion: characteristics, causes and conversion. Immunology. 2010;129(4):474-481.

13. Mazzoni A, et al. First-dose mRNA vaccination is sufficient to reactivate immunological memory to SARS-CoV-2 in subjects who have recovered from COVID-19. J Clin Invest. 2021;131(12):e149150.

14. Bonelli F, et al. Clinical and analytical performance of an automated serological test that identifies S1/S2 neutralizing IgG in COVID-19 patients semiquantitatively. JClin Microbiol. 2020;58(9):e01224-20.

15. Sandri MT, et al. SARS-CoV-2 serology in 4000 health care and administrative staff across seven sites in Lombardy, Italy [preprint]. https://doi. org/10.1101/2020.05.24.20111245. Posted on medRxiv December 2, 2020. 